\title{
CONGRUENCES ON ORTHODOX SEMIGROUPS WITH ASSOCIATE SUBGROUPS
}

\author{
by T. S. BLYTH, EMÍlIA GIRALDES and M. PAULA O. MARQUES-SMITH
}

(Received 12 September, 1994)

If $S$ is a regular semigroup then an inverse transversal of $S$ is an inverse subsemigroup $T$ with the property that $|T \cap V(x)|=1$ for every $x \in S$ where $V(x)$ denotes the set of inverses of $x \in S$. In a previous publication [1] we considered the similar concept of a subsemigroup $T$ of $S$ such that $|T \cap A(x)|=1$ for every $x \in S$ where $A(x)=\{y \in S ; x y x=$ $x$ \} denotes the set of associates (or pre-inverses) of $x \in S$, and showed that such a subsemigroup $T$ is necessarily a maximal subgroup $H_{\alpha}$ for some idempotent $\alpha \in S$. Throughout what follows, we shall assume that $S$ is orthodox and $\alpha$ is a middle unit (in the sense that $x \alpha y=x y$ for all $x, y \in S$ ). Under these assumptions, we obtained in [1] a structure theorem which generalises that given in [3] for uniquely unit orthodox semigroups. Adopting the notation of [1], we let $T \cap A(x)=\left\{x^{*}\right\}$ and write the subgroup $T$ as $H_{\alpha}=\left\{x^{*} ; x \in S\right\}$, which we call an associate subgroup of $S$. For every $x \in S$ we therefore have $x^{*} \alpha=x^{*}=\alpha x^{*}$ and $x^{*} x^{* *}=\alpha=x^{* *} x^{*}$. As shown in [1, Theorems 4, 5] we also have $(x y)^{*}=y^{*} x^{*}$ for all $x, y \in S$, and $e^{*}=\alpha$ for every idempotent $e$.

Our objective here is to consider congruences on such a semigroup. Since the building bricks in the structure theorem [1] are the subgroup $H_{a}$ and the sub-bands $\alpha E$, $E \alpha$ of the band $E$ of idempotents of $S$, these three subsemigroups will play an important rôle in what follows.

As we shall see, the study of congruences is intimately related to certain residuated mappings that arise naturally. We recall that if $A, B$ are ordered sets then a mapping $f: A \rightarrow B$ is said to be residuated if the pre-image of every principal down-set of $B$ is a principal down-set of $A$. For the general properties of residuated mappings we refer the reader to [2]. For our purposes here we require the fact that $f: A \rightarrow B$ is residuated if and only if it is isotone and there is a (necessarily unique) isotone mapping $f^{+}: B \rightarrow A$ such that $f^{+} \circ f \geqslant \mathrm{id}_{A}$ and $f \circ f^{+} \leqslant \mathrm{id}_{B}$.

Since, in the semigroups under consideration, the unary operation $x \mapsto x^{*}$ is significant, it is reasonable to expect that an important rôle will be played by the semigroup congruences $\vartheta$ such that

$$
(x, y) \in \vartheta \Rightarrow\left(x^{*}, y^{*}\right) \in \vartheta
$$

i.e. the congruences on the algebra $\left(S, .,^{*}\right)$ which we shall refer to as *-congruences. We shall denote by Con $S$ the complete lattice of (semigroup) congruences on $S$. It is easily seen that the set of ${ }^{*}$-congruences forms a complete sublattice of Con $S$; we denote this by Con*S.

Definition. Let $\lambda, \pi, \mu$ be congruences on $\alpha E, H_{\alpha}, E \alpha$ respectively. We shall call the triple $(\lambda, \pi, \mu)$ weighted if there exists $\Theta \in \operatorname{Con} S$ such that

(a) $\left.\Theta\right|_{H_{\mathrm{a}}}=\pi$

(b) $\left.\lambda\right|_{\alpha E \alpha}=\left.\mu\right|_{\alpha E \alpha}=\left.\Theta\right|_{\alpha E \alpha}$.

Glasgow Math. J. 38 (1996) 113-124. 
We shall denote by WT $(S)$ the set of weighted triples on $S$. Given $(\lambda, \pi, \mu) \in \mathrm{WT}(S)$ let $\Psi(\lambda, \pi, \mu)$ be the relation on $S$ given by

$$
(x, y) \in \Psi(\lambda, \pi, \mu) \Leftrightarrow\left(x^{*} x, y^{*} y\right) \in \lambda,\left(x^{*}, y^{*}\right) \in \pi,\left(x x^{*}, y y^{*}\right) \in \mu .
$$

THEOREM 1. If $(\lambda, \pi, \mu) \in \mathrm{WT}(S)$ then $\Psi(\lambda, \pi, \mu) \in$ Con*S.

Proof. Suppose that $\Theta \in \operatorname{Con} S$ satisfies (a), (b) and let $(x, y) \in \Psi(\lambda, \pi, \mu)$. Then $\left(x^{*}, y^{*}\right) \in \pi$ and so, by (a), we have $\left(x^{*}, y^{*}\right) \in \Theta$ and $\left(x^{* *}, y^{* *}\right) \in \Theta$. It follows that, for every $z \in S$,

$$
\left(x^{* *} z z^{*} x^{*}, y^{* *} z z^{*} y^{*}\right) \in \Theta .
$$

Since each side of this belongs to $\alpha E \alpha$ we then have, by (b),

$$
\left(x^{* *} z z^{*} x^{*}, y^{* *} z z^{*} y^{*}\right) \in \mu \text {. }
$$

Now

$$
x z(x z)^{*}=x z z^{*} x^{*}=x \alpha z z^{*} x^{*}=x x^{*} \cdot x^{* *} z z^{*} x^{*}
$$

and so, by $\left(^{*}\right)$ and the fact that $\left(x x^{*}, y y^{*}\right) \in \mu$, we deduce that

$$
\left(x z(x z)^{*}, y z(y z)^{*}\right) \in \mu .
$$

Observe also that $\left(x^{*} x, y^{*} y\right) \in \lambda$ gives

$$
\left.\left(x^{*} x \alpha, y^{*} y \alpha\right) \in \lambda\right|_{\alpha E \alpha}=\left.\Theta\right|_{\alpha E \alpha}
$$

whence, for every $z \in S$,

$$
\left.\left(z^{*} x^{*} x z^{* *}, z^{*} y^{*} y z^{* *}\right) \in \Theta\right|_{\alpha E \alpha}=\left.\lambda\right|_{\alpha E \alpha}
$$

and consequently

$$
\left(z^{*} x^{*} x z^{* *} z^{*} z, z^{*} y^{*} y z^{* *} z^{*} z\right) \in \lambda,
$$

which reduces to $\left((x z)^{*} x z,(y z)^{*} y z\right) \in \lambda$. Since clearly $\left((x z)^{*},(y z)^{*}\right)=\left(z^{*} x^{*}, z^{*} y^{*}\right) \in \pi$ it follows that $(x z, y z) \in \Psi(\lambda, \pi, \mu)$. In a similar way we can show that $(z x, z y) \in$ $\Psi(\lambda, \pi, \mu)$. Hence $\Psi(\lambda, \pi, \mu)$ is a congruence.

Now $x^{*} x^{* *}=\alpha=x^{* *} x^{*}$ for every $x \in S$ and, since $\pi$ is a group congruence, we have

$$
\left(x^{*}, y^{*}\right) \in \pi \Rightarrow\left(x^{* *}, y^{* *}\right)=\left(\left(x^{*}\right)^{-1},\left(y^{*}\right)^{-1}\right) \in \pi \text {. }
$$

It follows that $\left(x^{*}, y^{*}\right) \in \Psi(\lambda, \pi, \mu)$ and therefore $\Psi(\lambda, \pi, \mu)$ is a ${ }^{*}$-congruence.

In what follows we shall assume that the set WT(S) of weighted triples on $S$ is given the cartesian order.

THEOREM 2. The mapping $\Psi: \mathrm{WT}(S) \rightarrow \operatorname{Con} S$ is injective and residuated, with residual $\Psi^{+}$given by

$$
\Psi^{+}(\vartheta)=\left(\left.\vartheta\right|_{\alpha E},\left.\vartheta\right|_{H_{\alpha}},\left.\vartheta\right|_{E \alpha}\right)
$$

Moreover, WT(S) is a lattice that is isomorphic to Con* $S$.

Proof. With notation as above, observe that

$$
\left(x^{*}, y^{*}\right) \in \Psi(\lambda, \pi, \mu) \Leftrightarrow\left(x^{* *}, y^{* *}\right) \in \pi \Leftrightarrow\left(x^{*}, y^{*}\right) \in \pi,
$$

and that therefore $\left.\Psi(\lambda, \pi, \mu)\right|_{H_{\alpha}}=\pi$. 
Also, for $e \alpha \in E \alpha$ we have

$$
(e \alpha)^{*}=\alpha^{*} e^{*}=\alpha \alpha=\alpha, \quad(e \alpha)^{*} e \alpha=\alpha e \alpha \in \alpha E \alpha, \quad e \alpha(e \alpha)^{*}=e \alpha .
$$

These equalities, together with (b), show that

$$
(e \alpha, f \alpha) \in \Psi(\lambda, \pi, \mu) \Leftrightarrow(e \alpha, f \alpha) \in \mu
$$

and that therefore $\left.\Psi(\lambda, \pi, \mu)\right|_{E \alpha}=\mu$. Similarly, $\left.\Psi(\lambda, \pi, \mu)\right|_{\alpha E}=\lambda$.

It follows from these observations that

$$
\Psi^{+} \Psi(\lambda, \pi, \mu)=(\lambda, \pi, \mu) .
$$

Moreover, using the identity $x=x x^{*} x^{* *} x * x$ and the fact that $\left.\vartheta\right|_{H_{\alpha}}$ is a group congruence, we have that

$$
\begin{aligned}
(x, y) \in \Psi \Psi^{+}(\vartheta) & \left.\Rightarrow\left(x^{*} x, y^{*} y\right) \in \vartheta\right|_{\alpha E},\left.\left(x^{*}, y^{*}\right) \in \vartheta\right|_{H_{\alpha}},\left.\left(x x^{*}, y y^{*}\right) \in \vartheta\right|_{E \alpha} \\
& \Rightarrow(x, y)=\left(x x^{*} x^{* *} x^{*} x, y y^{*} y^{* *} y^{*} y\right) \in \vartheta
\end{aligned}
$$

and therefore

$$
\Psi \Psi^{+}(\vartheta) \subseteq \vartheta
$$

Since both $\Psi$ and $\Psi^{+}$are isotone, it follows from (1) and (2) that $\Psi$ is injective and residuated, with residual $\Psi^{+}$.

By. Theorem 1 we have that $\operatorname{Im} \Psi \subseteq \operatorname{Con}^{*} S$. Conversely, if $\vartheta \in \operatorname{Con}^{*} S$ then $(x, y) \in \vartheta$ gives $\left(x^{*} x, y^{*} y\right) \in \vartheta,\left(x^{*}, y^{*}\right) \in \vartheta,\left(x x^{*}, y y^{*}\right) \in \vartheta$ whence $(x, y) \in \Psi \Psi^{+}(\vartheta)$. Thus $\vartheta \subseteq \Psi \Psi^{+}(\vartheta)$ and it follows from (2) that $\vartheta=\Psi \Psi^{+}(\vartheta) \in \operatorname{Im} \Psi$. Consequently, $\operatorname{Im} \Psi=$ Con* $S$. Now since $\Psi$ is isotone and injective it induces an isotone bijection $\Psi_{*}: \mathrm{WT}(S) \rightarrow \operatorname{Im} \Psi$. It follows from the above that the restriction of $\Psi^{+}$to Con* $S$ is isotone and is the inverse of $\Psi_{*}$. Consequently, $W T(S) \simeq \operatorname{Im} \Psi$. It follows from these observations that WT $(S)$ is isomorphic to the lattice Con* $S$.

COROLlary 1. The relation $\equiv$ defined on Con $S$ by

$$
\left.\vartheta \equiv \varphi \Leftrightarrow \vartheta\right|_{\alpha E}=\left.\varphi\right|_{\alpha E},\left.\quad \vartheta\right|_{H_{\alpha}}=\left.\varphi\right|_{H_{a}},\left.\quad \vartheta\right|_{E \alpha}=\left.\varphi\right|_{E \alpha}
$$

is a dual closure equivalence. The smallest element in the $\equiv$-class of $\vartheta$ is $\Psi \Psi^{+}(\vartheta)$.

Proof. Observe that, since $\Psi$ is residuated, $\Psi \Psi^{+}$is a dual closure; and, since $\Psi^{+} \Psi \Psi^{+}=\Psi^{+}$,

$$
\vartheta \equiv \varphi \Leftrightarrow \Psi^{+}(\vartheta)=\Psi^{+}(\varphi) \Leftrightarrow \Psi \Psi^{+}(\vartheta)=\Psi \Psi^{+}(\varphi) .
$$

Corollary 2. There is a lattice isomorphism Con* $S \simeq($ Con $S) / \equiv$.

Corollary 3. If $(\lambda, \pi, \mu),\left(\lambda^{\prime}, \pi^{\prime}, \mu^{\prime}\right) \in \mathrm{WT}(S)$ then

$$
\begin{aligned}
& \Psi(\lambda, \pi, \mu) \cap \Psi\left(\lambda^{\prime}, \pi^{\prime}, \mu^{\prime}\right)=\Psi\left(\lambda \cap \lambda^{\prime}, \pi \cap \pi^{\prime}, \mu \cap \mu^{\prime}\right) ; \\
& \Psi(\lambda, \pi, \mu) \vee \Psi\left(\lambda^{\prime}, \pi^{\prime}, \mu^{\prime}\right)=\Psi\left(\lambda \vee \lambda^{\prime}, \pi \vee \pi^{\prime}, \mu \vee \mu^{\prime}\right) .
\end{aligned}
$$

Proof. This follows immediately from the fact that $(\lambda, \pi, \mu) \mapsto \Psi(\lambda, \pi, \mu)$ is a lattice isomorphism from WT $(S)$ to Con* $S$.

We now consider the extension of congruences on $\alpha E, H_{\alpha}, E \alpha$ to ${ }^{*}$-congruences on 
$S$. We begin with the subgroup $H_{\alpha}$. For this purpose we shall use the notation $\iota_{X}$ to denote the universal congruence on a given subset $X$ of $S$.

Theorem 3. For every $\vartheta \in \operatorname{Con} H_{\alpha}$ the relation $\hat{\vartheta}$ defined on $S$ by

$$
(a, b) \in \hat{\vartheta} \Leftrightarrow\left(a^{*}, b^{*}\right) \in \vartheta
$$

is the biggest *-congruence on $S$ whose restriction to $H_{\alpha}$ is $\vartheta$. The weighted triple that corresponds to $\hat{\vartheta}$ is $\left(\iota_{\alpha E}, \vartheta, \iota_{E \alpha}\right)$.

Proof. If $(a, b) \in \hat{\vartheta}$ then $\left(a^{*}, b^{*}\right) \in \vartheta$ and therefore, since $\vartheta$ is a congruence on the group $H_{\alpha}$, we have $\left(a^{*} x^{*}, b^{*} x^{*}\right) \in \vartheta$ for every $x \in S$. Since $a^{*} x^{*}=(x a)^{*}$ it follows that $(x a, x b) \in \hat{\vartheta}$. Similarly, $(a x, b x) \in \hat{\vartheta}$ and so $\hat{\vartheta} \in \operatorname{Con} S$. Since $\left(a^{*}, b^{*}\right) \in \vartheta$ implies $\left(a^{* *}, b^{* *}\right) \in \vartheta$ it follows that $\hat{\vartheta}$ is a ${ }^{*}$-congruence. Now if $a, b \in H_{\alpha}$ we have that

$$
\left.(a, b) \in \hat{\vartheta}\right|_{H_{\alpha}} \Leftrightarrow\left(a^{*}, b^{*}\right) \in \vartheta \Leftrightarrow(a, b)=\left(a^{* *}, b^{* *}\right) \in \vartheta
$$

and consequently we see that $\left.\hat{\vartheta}\right|_{H_{\mathrm{a}}}=\vartheta$. If now $\varphi$ is a ${ }^{*}$-congruence such that $\left.\varphi\right|_{H_{\mathrm{o}}}=\vartheta$ then

$$
\left.(a, b) \in \varphi \Rightarrow\left(a^{*}, b^{*}\right) \in \varphi\right|_{H_{\mathrm{a}}}=\vartheta \Rightarrow(a, b) \in \hat{\vartheta}
$$

so that $\varphi \subseteq \hat{\vartheta}$. Since $e^{*}=\alpha$ for every $e \in \alpha E \cup E \alpha$ is clear that $\left.\hat{\vartheta}\right|_{\alpha E}=\iota_{\alpha E}$ and $\left.\hat{\vartheta}\right|_{E \alpha}=\iota_{E \alpha}$. It follows that the weighted triple that corresponds to $\hat{\vartheta}$ is $\Psi^{+}(\hat{\vartheta})=\left(\iota_{\alpha E}, \vartheta, \iota_{E \alpha}\right)$.

Corollary. Every $\vartheta \in \operatorname{Con} H_{\alpha}$ is the middle component of some weighted triple. The biggest *-congruence that corresponds to a weighted triple of the form $(-, \vartheta,-)$ is $\hat{\vartheta}$.

Theorem 4. The mapping $\Omega$ :Con* $S \rightarrow$ Con $H_{\alpha}$ given by $\Omega(\varphi)=\left.\varphi\right|_{H_{\alpha}}$ is surjective and residuated, with residual $\Omega^{+}$given by $\Omega^{+}(\vartheta)=\hat{\vartheta}$.

Proof. Each of $\Omega$ and $\Omega^{+}$as defined is isotone and, for all $\varphi \in$ Con* $S$ and all $\vartheta \in \operatorname{Con} H_{\alpha}$,

$$
\begin{aligned}
& \Omega^{+} \Omega(\varphi)=\Omega^{+}\left(\left.\varphi\right|_{H_{\mathrm{a}}}\right)=\widehat{\left.\varphi\right|_{H_{\mathrm{a}}}} \geqslant \varphi ; \\
& \Omega \Omega^{+}(\vartheta)=\Omega(\hat{\vartheta})=\left.\hat{\vartheta}\right|_{H_{\mathrm{a}}}=\vartheta .
\end{aligned}
$$

Corollary. Con $H_{\alpha}$ is isomorphic to the sublattice of Con* $S$ consisting of those *-congruences $\varphi$ such that $\left.\varphi\right|_{\alpha E}=\iota_{\alpha E}$ and $\left.\varphi\right|_{E \alpha}=\iota_{E \alpha}$.

Proof. Since $\Omega^{+}$is injective the sublattice in question is $\operatorname{Im} \Omega^{+}$. Now we have $\Omega^{+} \Omega \Omega^{+}=\Omega^{+}$and so $\operatorname{Im} \Omega^{+}=\operatorname{Im} \Omega^{+} \Omega$, which consists of those ${ }^{*}$-congruences $\varphi$ such that $\varphi=\widehat{\left.\varphi\right|_{H_{\mathrm{a}}}}$. The conclusion follows from Theorem 3 .

We now consider extensions of congruences on $\alpha E$ and $E \alpha$. For this purpose we require the fact that if $e \in E$ then for every $x \in S$ we have $x^{*} e x \in E$ and $x e x^{*} \in E$. Indeed, by [1, Corollary 1 of Theorem 5] we have that $e^{*}=\alpha$ for every $e \in E$ and therefore $x^{*} e x=x^{*} \alpha e x=x^{*} e^{*} e x=(e x)^{*} e x \in E$ and similarly $x e x^{*} \in E$.

Definition. We shall say that $\lambda \in \operatorname{Con} \alpha E$ is special if, for all $e, f \in \alpha E$,

$$
(e, f) \in \lambda \Rightarrow(\forall x \in S) \quad\left(x^{*} e x, x^{*} f x\right) \in \lambda .
$$


Similarly, $\mu \in \operatorname{Con} E \alpha$ will be called special if, for all $e, f \in E \alpha$,

$$
(e, f) \in \mu \Rightarrow(\forall x \in S) \quad\left(x e x^{*}, x f x^{*}\right) \in \mu .
$$

In what follows, given $\lambda \in$ Con $\alpha E$ we shall be interested in the relation $\bar{\lambda}$ defined on $S$ by

$$
(a, b) \in \bar{\lambda} \Leftrightarrow(\forall e \in R) \quad\left(a^{*} e a, b^{*} e b\right) \in \lambda ;
$$

and dually, for $\mu \in \operatorname{Con} E \alpha$, the relation $\bar{\mu}$ defined on $S$ by

$$
(a, b) \in \bar{\mu} \Leftrightarrow(\forall e \in E) \quad\left(a e a^{*}, b e b^{*}\right) \in \mu .
$$

Note that $\bar{\lambda}$ is a left congruence on $S$; for if $(a, b) \in \bar{\lambda}$ then, since $x^{*} e x \in E$ for every $x \in S$, we have $\left(a^{*} x^{*} e x a, b^{*} x^{*} e x b\right) \in \lambda$, so that $(x a, x b) \in \bar{\lambda}$. Similarly, $\bar{\mu}$ is a right congruence.

THEOREM 5. For $\lambda \in$ Con $\alpha E$ the following statements are equivalent:

(1) $\lambda$ is special;

(2) $\lambda=\left.\vartheta\right|_{\alpha E}$ for some $\vartheta \in \operatorname{Con} S$.

Proof. (1) $\Rightarrow$ (2). Suppose that $\lambda$ is special. Then we have $\bar{\lambda} \in \operatorname{Con} S$. For, if $(a, b) \in \bar{\lambda}$ then, since $\lambda$ is special and $a^{*} e a \in \alpha E$, for every $x \in S$ we have $\left(x^{*} a^{*} e a x, x^{*} b^{*} e b x\right) \in \lambda$, so that $(a x, b x) \in \bar{\lambda}$, whence $\bar{\lambda}$ is a right congruence. As observed above, $\bar{\lambda}$ is a left congruence.

If now $f, g \in \alpha E$ are such that $(f, g) \in \bar{\lambda}$ then since $f^{*}=g^{*}=\alpha$ we have $(\alpha e f, \alpha e g) \in$ $\lambda$ for every $e \in E$. Taking $e=\alpha$ we obtain $(f, g) \in \lambda$. Conversely, if $(f, g) \in \lambda$ then $(\alpha e f, \alpha e g) \in \lambda$ gives $\left(f^{*} e f, g^{*} e g\right) \in \lambda$ whence $(f, g) \in \bar{\lambda}$. Hence $\left.\bar{\lambda}\right|_{\alpha E}=\lambda$, so (2) holds with $\vartheta=\bar{\lambda}$.

(2) $\Rightarrow(1)$. If $\vartheta \in \operatorname{Con} S$ then for $e, f \in \alpha E$ we have

$$
\left.(e, f) \in \vartheta\right|_{\alpha E} \Rightarrow(e, f) \in \vartheta \Rightarrow(\forall x \in S)\left(x^{*} e x, x^{*} f x\right) \in \vartheta
$$

Since $x^{*} e x \in \alpha E$, it follows that $\left.\vartheta\right|_{\alpha E}$ is special.

COROLLARY 1. For every special congruence $\lambda$ on $\alpha E$ there is a biggest *-congruence $\vartheta$ on $S$ such that $\left.\vartheta\right|_{\alpha E}=\lambda$, namely $\vartheta=\Psi \Psi^{+}(\bar{\lambda})$.

Proof. By Theorem 2, $\Psi \Psi^{+}(\bar{\lambda}) \in$ Con* $S$ with $\left.\Psi \Psi^{+}(\bar{\lambda})\right|_{\alpha E}=\left.\bar{\lambda}\right|_{\alpha E}=\lambda$. If now $\zeta \in$ Con* $S$ is such that $\left.\zeta\right|_{\alpha E}=\lambda$ then

$$
\left.(a, b) \in \zeta \Rightarrow(\forall e \in E)\left(a^{*} e a, b^{*} e b\right) \in \zeta\right|_{\alpha E}=\lambda \Rightarrow(a, b) \in \bar{\lambda}
$$

so $\zeta \subseteq \vec{\lambda}$ and consequently $\zeta=\Psi \Psi^{+}(\zeta) \subseteq \Psi \Psi^{+}(\bar{\lambda})$.

COROLlaRY 2. The following statements concerning $\lambda \in \operatorname{Con} \alpha E$ are equivalent:

(1) $\lambda$ is special;

(2) there is a weighted triple whose first component is $\lambda$.

Proof. (1) $\Rightarrow(2)$. If (1) holds then the weighted triple associated with $\Psi \Psi^{+}(\bar{\lambda})$ has first component $\lambda$. have

$(2) \Rightarrow(1)$. If there is a weighted triple of the form $(\lambda,-,-)$ then by Theorem 2 we

$$
(\lambda,-,-)=\Psi^{+} \Psi(\lambda,-,-)
$$

whence $\lambda=\left.\Psi(\lambda,-,-)\right|_{\alpha E}$ and so $\lambda$ is special. 
Let $\operatorname{SpCon} \alpha E$ be the set of special congruences on $\alpha E$. It is immediate from Corollary 2 above and the fact that $\mathrm{WT}(S)$ is a lattice that $\operatorname{SpCon} \alpha E$ is a sublattice of Con $\alpha E$.

THEOREM 6. The mapping $\Phi_{\alpha E}$ :Con* $S \rightarrow \operatorname{SpCon} \alpha E$ given by $\Phi_{\alpha E}(\vartheta)=\left.\vartheta\right|_{\alpha E}$ is surjective and residuated, with residual $\Phi_{\alpha E}^{+}$given by $\Phi_{\alpha E}^{+}(\lambda)=\Psi \Psi^{+}(\bar{\lambda})$.

Proof. Each of $\Phi_{\alpha E}$ and $\Phi_{\alpha E}^{+}$as defined is isotone. For every $\vartheta \in$ Con* $S$ we have

$$
\Phi_{\alpha E}^{+} \Phi_{\alpha E}(\vartheta)=\Phi_{\alpha E}^{+}\left(\left.\vartheta\right|_{\alpha E}\right)=\Psi \Psi^{+}\left(\overline{\left.\vartheta\right|_{\alpha E}}\right) \geqslant \vartheta
$$

since, by the Corollary to Theorem $5, \Psi \Psi^{+}\left(\overline{\left.\vartheta\right|_{\alpha E}}\right)$ is the biggest *-congruence on $S$ whose restriction to $\alpha E$ is $\left.\vartheta\right|_{\alpha E}$. Also, for every $\lambda \in \operatorname{SpCon} \alpha E$ we have

$$
\Phi_{\alpha E} \Phi_{\alpha E}^{+}(\lambda)=\Phi_{\alpha E} \Psi \Psi^{+}(\bar{\lambda})=\left.\Psi \Psi^{+}(\bar{\lambda})\right|_{\alpha E}=\left.\bar{\lambda}\right|_{\alpha E}=\lambda .
$$

Hence $\Phi_{\alpha E}$ is surjective and residuated, with residual $\Phi_{\alpha E}^{+}$.

There is, of course, a dual to Theorem 6 that involves $E \alpha$.

THEOREM 7. Let $\lambda \in \operatorname{Con} \alpha E$ and $\mu \in \operatorname{Con} E \alpha$. Then the following statements are equivalent:

(1) $\lambda, \mu$ are the first and third components of some common weighted triple;

(2) $\lambda, \mu$ are special and $\left.\lambda\right|_{\alpha E_{\alpha}}=\left.\mu\right|_{\alpha E_{\alpha}}$.

Proof. (1) $\Rightarrow(2)$. If $(\lambda, \pi, \mu) \in \mathrm{WT}(S)$ then $\lambda$ and $\mu$ are special by Corollary 2 of Theorem 5 and, from the definition of weighted triple, $\left.\lambda\right|_{\alpha E \alpha}=\left.\mu\right|_{\alpha E \alpha \text {. }}$.

(2) $\Rightarrow(1)$. Observe that since $\bar{\lambda} \in \operatorname{Con} S$ and $\lambda=\left.\bar{\lambda}\right|_{\alpha E}$ we have, for all $p, q \in E$,

$$
\begin{aligned}
(\alpha p, \alpha q) \in \lambda & \Rightarrow(\alpha p, \alpha q) \in \bar{\lambda} \\
& \left.\Rightarrow(\forall e \in E) \quad(\alpha p e \alpha, \alpha q e \alpha) \in \bar{\lambda}\right|_{\alpha E \alpha}=\left.\lambda\right|_{\alpha E \alpha}=\left.\mu\right|_{\alpha E \alpha} \\
& \Rightarrow(\alpha p, \alpha q) \in \bar{\mu} .
\end{aligned}
$$

Consequently, $\left.\lambda \subseteq \bar{\mu}\right|_{\alpha E} ;$ and similarly $\left.\mu \subseteq \bar{\lambda}\right|_{E \alpha}$.

For $a, b \in H_{\alpha}$ we have

$$
\left.\left.(a, b) \in \bar{\lambda}\right|_{H_{\alpha}} \Leftrightarrow(\forall e \in E) \quad\left(a^{*} e a, b^{*} e b\right) \in \lambda\right|_{\alpha E \alpha}=\left.\left.\mu\right|_{\alpha E \alpha} \Leftrightarrow\left(a^{*}, b^{*}\right) \in \bar{\mu}\right|_{H_{\alpha}},
$$

this being equivalent to $\left.(a, b) \in \bar{\mu}\right|_{H_{\alpha}}$, so that $\left.\bar{\lambda}\right|_{H_{a}}=\left.\bar{\mu}\right|_{H_{a}}$.

Now, under the *-congruence/weighted triple bijection of Theorem 2 , we have

$$
\Psi \Psi^{+}(\bar{\lambda}) \sim\left(\lambda,\left.\bar{\lambda}\right|_{H_{\alpha}},\left.\bar{\lambda}\right|_{E_{\alpha}}\right), \quad \Psi \Psi^{+}(\bar{\mu}) \sim\left(\left.\bar{\mu}\right|_{\alpha E},\left.\bar{\mu}\right|_{H_{\alpha}}, \mu\right) .
$$

Defining $\pi_{\lambda, \mu}=\left.\bar{\lambda}\right|_{H_{a}}=\left.\bar{\mu}\right|_{H_{a}}$, we see that the *-congruence $\Psi \Psi^{+}(\bar{\lambda}) \cap \Psi \Psi^{+}(\bar{\mu})$ corresponds to the weighted triple $\left(\lambda, \pi_{\lambda, \mu}, \mu\right)$.

COROLlaRY 1 . There is a biggest *-congruence that corresponds to a weighted triple of the form $(\lambda,-, \mu)$, namely $\Psi \Psi^{+}(\bar{\lambda}) \cap \Psi \Psi^{+}(\bar{\mu})$.

Proof. For $(\lambda, \pi, \mu) \in \mathrm{WT}(S)$, let $\Theta \in \operatorname{Con} S$ satisfy (a), (b) and let $P=\Psi(\lambda, \pi, \mu)$. 
If $\left(a^{*}, b^{*}\right) \in \pi$ then we have $\left(a^{* *}, b^{* *}\right) \in \pi$ whence $\left(a^{*}, b^{*}\right) \in \Theta$ and $\left(a^{* *}, b^{* *}\right) \in \Theta$, from which it follows that

$$
\left.(\forall e \in E) \quad\left(a^{*} e a^{* *}, b^{*} e b^{* *}\right) \in \Theta\right|_{\alpha E \alpha}=\left.\lambda\right|_{\alpha E \alpha}
$$

and therefore $\left(a^{* *}, b^{* *}\right) \in \bar{\lambda}$.

Since $\lambda=\left.\bar{\lambda}\right|_{\alpha E}$ and since, as seen above, $\left.\mu \subseteq \bar{\lambda}\right|_{E \alpha}$ it follows that

$$
\begin{aligned}
(a, b) \in P & \Rightarrow\left(a^{*} a, b^{*} b\right) \in \bar{\lambda},\left(a^{* *}, b^{* *}\right) \in \bar{\lambda},\left(a a^{*}, b b^{*}\right) \in \bar{\lambda} \\
& \Rightarrow(a, b) \in \bar{\lambda}
\end{aligned}
$$

so $P \subseteq \bar{\lambda}$ and therefore $P=\Psi \Psi^{+}(P) \subseteq \Psi \Psi^{+}(\bar{\lambda})$. Arguing similarly with $\mu$, we obtain

$$
P \subseteq \Psi \Psi^{+}(\bar{\lambda}) \cap \Psi \Psi^{+}(\bar{\mu})
$$

from which the result follows.

COROLLARY 2. The biggest weighted triple of the form $(\lambda,-, \mu)$ has middle component $\pi_{\lambda, \mu}=\left.\bar{\lambda}\right|_{H_{a}}=\left.\bar{\mu}\right|_{H_{a}}$.

Given $\lambda \in \operatorname{SpCon} \alpha E$ and $\mu \in \operatorname{SpCon} E \alpha$, let $T_{\lambda, \mu}$ be the set of weighted triples with first component $\lambda$ and third component $\mu$. As in the proof of Theorem 7, let $\pi_{\lambda, \mu}=\left.\bar{\lambda}\right|_{H_{o}}=\left.\bar{\mu}\right|_{H_{a}}$.

THEOREM 8. $T_{\lambda, \mu}$ is a sublattice of $\mathrm{WT}(S)$, isomorphic to the interval $\left[\omega, \pi_{\lambda, \mu}\right]$ of Con $H_{\alpha}$.

Proof. Clearly, $T_{\lambda, \mu}$ is a sublattice of WT(S). If now $(\lambda, \vartheta, \mu) \in T_{\lambda, \mu}$ then by Corollary 2 of Theorem 7 we have $\vartheta \subseteq \pi_{\lambda, \mu}$. Consider the mapping $\zeta: T_{\lambda, \mu} \rightarrow\left[\omega, \pi_{\lambda, \mu}\right]$ given by $\zeta(\lambda, \vartheta, \mu)=\vartheta$. Clearly, $\zeta$ is an injective $\cap$-morphism. It suffices, therefore, to prove that $\zeta$ is surjective; equivalently, that if $\vartheta \in\left[\omega, \pi_{\lambda, \mu}\right]$ then $(\lambda, \vartheta, \mu)$ is a weighted triple. Now, by Theorem $3, \hat{\vartheta}$ is represented by the weighted triple

$$
\left(\iota_{\alpha E}, \vartheta, \iota_{E \alpha}\right) \text {, }
$$

whereas $\Psi \Psi^{+}(\bar{\lambda})$ is represented by the weighted triple

$$
\left(\lambda, \pi_{\lambda, \mu},\left.\bar{\lambda}\right|_{E \alpha}\right)
$$

and $\Psi \Psi^{+}(\bar{\mu})$ is represented by the weighted triple

$$
\left(\left.\bar{\mu}\right|_{\alpha E}, \pi_{\lambda, \mu}, \mu\right) \text {. }
$$

It follows by Theorem 2 that the *-congruence $\hat{\vartheta} \cap \Psi \Psi^{+}(\bar{\lambda}) \cap \Psi \Psi^{+}(\bar{\mu})$ is represented by the intersection of these three triples which, since $\left.\lambda \subseteq \bar{\mu}\right|_{\alpha E}$ and $\left.\mu \subseteq \bar{\lambda}\right|_{E \alpha \alpha}$, is $(\lambda, \vartheta, \mu)$.

Corollary. For every $(\lambda, \vartheta, \mu) \in \mathrm{WT}(S)$ we have

$$
\Psi(\lambda, \vartheta, \mu)=\hat{\vartheta} \cap \Psi \Psi^{+}(\bar{\lambda}) \cap \Psi \Psi^{+}(\bar{\mu}) .
$$

Given $\varphi \in$ Con $\alpha E$ consider now the relation $\lambda_{\varphi}$ defined on $\alpha E$ by

$$
(a, b) \in \lambda_{\varphi} \Leftrightarrow(a \alpha, b \alpha) \in \varphi
$$


Clearly, if $(a, b) \in \lambda_{\varphi}$ then $(x a, x b) \in \lambda_{\varphi}$ for all $x \in \alpha E$; and $a x \alpha=a \alpha x \alpha$ gives $(a x, b x) \in$ $\lambda_{\varphi}$. Thus $\lambda_{\varphi} \in$ Con $\alpha E$ with, clearly, $\left.\lambda_{\varphi}\right|_{\alpha E \alpha}=\varphi$. Similarly, if $\mu_{\varphi}$ is defined on $E \alpha$ by

$$
(a, b) \in \mu_{\varphi} \Leftrightarrow(\alpha a, \alpha b) \in \varphi
$$

then $\mu_{\varphi} \in \operatorname{Con} E \alpha$ with $\left.\mu_{\varphi}\right|_{\alpha E \alpha}=\varphi$.

THEOREM 9. The mapping $\delta_{\alpha E}$ :Con $\alpha E \rightarrow \operatorname{Con} \alpha E \alpha$ given by $\delta_{\alpha E}(\vartheta)=\left.\vartheta\right|_{\alpha E \alpha}$ is surjective and residuated, with residual $\delta_{\alpha E}^{+}$given by $\delta_{\alpha E}^{+}(\varphi)=\lambda_{\varphi}$.

Proof. Both $\delta_{\alpha E}$ and $\delta_{\alpha E}^{+}$as defined are isotone. Now, for every $\varphi \in$ Con $\alpha E \alpha$,

$$
\delta_{\alpha E} \delta_{\alpha E}^{+}(\varphi)=\delta_{\alpha E}\left(\lambda_{\varphi}\right)=\left.\lambda_{\varphi}\right|_{\alpha E \alpha}=\varphi .
$$

Also, if $\vartheta \in \operatorname{Con} \alpha E$ then

$$
\left.(a, b) \in \vartheta \Rightarrow(a \alpha, b \alpha) \in \vartheta\right|_{\alpha E \alpha} \Rightarrow(a, b) \in \lambda_{\left.\vartheta\right|_{\alpha F a}}
$$

so that $\vartheta \subseteq \lambda_{l_{\alpha E a}}$. Consequently,

$$
\delta_{\alpha E}^{+} \delta_{\alpha E}(\vartheta)=\delta_{\alpha E}^{+}\left(\left.\vartheta\right|_{\alpha E \alpha}\right)=\lambda_{\left.\vartheta\right|_{\alpha F \mathrm{a}}} \supseteq \vartheta
$$

Hence $\delta_{\alpha E}$ is surjective and residuated, with residual $\delta_{\alpha E}^{+}$.

We shall say that $\varphi \in$ Con $\alpha E \alpha$ is special if

$$
(a, b) \in \varphi \Rightarrow(\forall x \in S) \quad\left(x^{*} a x \alpha, x^{*} b x \alpha\right) \in \varphi, \quad\left(\alpha x a x^{*}, \alpha x b x^{*}\right) \in \varphi .
$$

THEOREM 10. The following statements concerning $\varphi \in \operatorname{Con} \alpha E \alpha$ are equivalent:

(1) $\varphi$ is special;

(2) $\lambda_{\varphi}$ and $\mu_{\varphi}$ are special;

(3) there is a weighted triple of the form $\left(\lambda_{\varphi},-, \mu_{\varphi}\right)$.

Proof. (1) $\Rightarrow$ (2). Suppose that (1) holds. Then

$$
\begin{aligned}
(e, f) \in \lambda_{\varphi} \Rightarrow(e \alpha, f \alpha) \in \varphi & \Rightarrow(\forall x \in S) & & \left(x^{*} e x \alpha, x^{*} f x \alpha\right) \in \varphi \\
& \Rightarrow(\forall x \in S) & & \left(x^{*} w x, x^{*} f x\right) \in \lambda_{\varphi},
\end{aligned}
$$

so $\lambda_{\varphi}$ is special. Similarly, so is $\mu_{\varphi}$.

(2) $\Rightarrow(1)$. If $\lambda_{\varphi}$ and $\mu_{\varphi}$ are special then

$$
\begin{aligned}
(e, f) \in \varphi=\left.\lambda_{\varphi}\right|_{\alpha E \alpha} & \Rightarrow(\forall x \in S) & \left(x^{*} e x, x^{*} f x\right) \in \lambda_{\varphi} \\
& \Rightarrow(\forall x \in S) & \left.\left(x^{*} e x \alpha, x^{*} f x \alpha\right) \in \lambda_{\varphi}\right|_{\alpha E \alpha}=\varphi,
\end{aligned}
$$

and similarly with $\mu_{\varphi}$. Hence $\varphi$ is special.

(2) $\Leftrightarrow$ (3). Since $\left.\lambda_{\varphi}\right|_{\alpha E \alpha}=\left.\mu_{\varphi}\right|_{\alpha E \alpha}=\varphi$, this follows by Theorem 7 .

If $\varphi_{1}, \varphi_{2} \in \operatorname{Con} \alpha E \alpha$ then it is readily seen that $\lambda_{\varphi_{1}} \cap \lambda_{\varphi_{2}}=\lambda_{\varphi_{1} \cap \varphi_{2}}$. Now, with $\equiv$ indicating $\lambda_{\varphi_{1}}$ or $\lambda_{\varphi_{2}}$ and $\sim$ indicating, as appropriate, $\varphi_{1}$ or $\varphi_{2}$, we have

$$
\begin{aligned}
(a, b) \in \lambda_{\varphi_{1}} \vee \lambda_{\varphi_{2}} & \Leftrightarrow a \equiv x_{1} \equiv x_{2} \equiv \ldots \equiv x_{n} \equiv b \\
& \Leftrightarrow a \alpha \sim x_{1} \alpha \sim x_{2} \alpha \sim \ldots \sim x_{n} \alpha \sim b \alpha \\
& \Leftrightarrow(a \alpha, b \alpha) \in \varphi_{1} \vee \varphi_{2} \\
& \Leftrightarrow(a, b) \in \lambda_{\varphi_{1} \vee \varphi_{2}}
\end{aligned}
$$


and therefore $\lambda_{\varphi_{1}} \vee \lambda_{\varphi_{2}}=\lambda_{\varphi_{1} \vee \varphi_{2}}$. Similarly we have $\mu_{\varphi_{1}} \cap \mu_{\varphi_{2}}=\mu_{\varphi_{1} \cap \varphi_{2}}$ and $\mu_{\varphi_{1}} \vee \mu_{\varphi_{2}}=$ $\mu_{\varphi_{1} \vee \varphi_{2}}$. It follows by Theorem 10 that if $\varphi_{1}, \varphi_{2} \in \operatorname{Con} \alpha E \alpha$ are special then so also are $\varphi_{1} \cap \varphi_{2}$ and $\varphi_{1} \vee \varphi_{2}$. Hence the set of special congruences on $\alpha E \alpha$ forms a lattice which we shall denote by $\operatorname{SpCon} \alpha E \alpha$.

Note now that if $\lambda \in \operatorname{SpCon} \alpha E$ then $\left.\lambda\right|_{\alpha E \alpha} \in \operatorname{SpCon} \alpha E \alpha$. In fact,

$$
\begin{aligned}
(a, b) \in \lambda & \Rightarrow(a \alpha, b \alpha) \in \lambda \\
& \Rightarrow(\forall x \in S) \quad\left(x^{*} a \alpha x, x^{*} b \alpha x\right) \in \lambda \\
& \left.\Rightarrow(\forall x \in S) \quad\left(x^{*} a x \alpha, x^{*} b x \alpha\right) \in \lambda\right|_{\alpha E \alpha},
\end{aligned}
$$

and

$$
\begin{aligned}
& \left.(a, b) \in \lambda \Rightarrow(\forall x \in S) \quad\left(x^{* *} a x^{*}, x^{* *} b x^{*}\right) \in \lambda\right|_{\alpha E \alpha}=\left.\mu\right|_{\alpha E \alpha} \\
& \Rightarrow(\forall x \in S) \quad\left(x a x^{*}, x b x^{*}\right)=\left(x x^{*} x^{* *} a x^{*}, x x^{*} x^{* *} b x^{*}\right) \in \mu \\
& \left.\Rightarrow(\forall x \in S) \quad\left(\alpha x a x^{*}, \alpha x b x^{*}\right) \in \mu\right|_{\alpha E \alpha}=\left.\lambda\right|_{\alpha E \alpha} \text {. }
\end{aligned}
$$

This observation, together with Theorems 9 and 10, gives immediately the following result.

THEOREM 11. The mapping $\Delta_{\alpha E}:$ SpCon $\alpha E \rightarrow \operatorname{SpCon} \alpha E \alpha$ given by $\Delta_{\alpha E}(\vartheta)=\left.\vartheta\right|_{\alpha E \alpha}$ is surjective and residuated, with residual $\Delta_{\alpha E}^{+}$given by $\Delta_{\alpha E}^{+}(\varphi)=\lambda_{\varphi}$.

Note also that if $\vartheta \in \operatorname{Con} S$ then $\left.\vartheta\right|_{\alpha E \alpha}$ is special. In fact, by Theorem $5,\left.\vartheta\right|_{\alpha E}$ is special and therefore, by the above observation, so is $\left.\vartheta\right|_{\alpha E \alpha}$. We can therefore use Theorems 6 and 11, and their duals to obtain the following result.

THEOREM 12. The mapping $\Gamma: \operatorname{Con} * S \rightarrow \operatorname{SpCon} \alpha E \alpha$ given by $\Gamma(\vartheta)=\left.\vartheta\right|_{a E \alpha}$ is surjective and residuated, with residual $\Gamma^{+}$given by

$$
\Gamma^{+}(\varphi)=\Psi\left(\lambda_{\varphi}, \pi_{\lambda_{\varphi}, \mu_{\varphi}}, \mu_{\varphi}\right)
$$

Proof. Consider the diagram

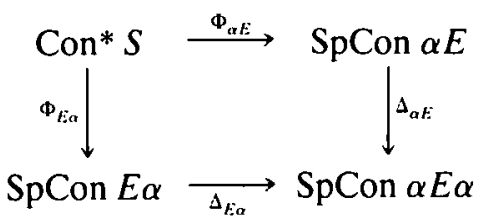

which is commutative since, by definition, $\Delta_{\alpha E} \Phi_{\alpha E}=\Gamma=\Delta_{E \alpha} \Phi_{E \alpha}$. That $\Gamma$ is surjective follows from Theorems 6 and 11. By [2, Theorem 2.8] we have on the one hand, for every $\varphi \in \operatorname{SpCon} \alpha E \alpha$,

$$
\Gamma^{+}(\varphi)=\Phi_{\alpha E}^{+} \Delta_{\alpha E}^{+}(\varphi)=\Phi_{\alpha E}^{+}\left(\lambda_{\varphi}\right)=\Psi \Psi^{+}\left(\overline{\lambda_{\varphi}}\right)=\Psi\left(\left.\overline{\varphi_{\varphi}}\right|_{\alpha E},\left.\overline{\lambda_{\varphi}}\right|_{H_{\alpha}},\left.\overline{\lambda_{\varphi}}\right|_{E \alpha}\right)
$$

and, on the other,

$$
\Gamma^{+}(\varphi)=\Phi_{E \alpha}^{+} \Delta_{E \alpha}^{+}(\varphi)=\Phi_{E \alpha}^{+}\left(\mu_{\varphi}\right)=\Psi \Psi^{+}\left(\overline{\mu_{\varphi}}\right)=\Psi\left(\left.\overline{\mu_{\varphi}}\right|_{\alpha E},\left.\overline{\mu_{\varphi}}\right|_{H_{\alpha}},\left.\overline{\mu_{\varphi}}\right|_{E \alpha}\right) .
$$

Since $\left.\overline{\lambda_{\varphi}}\right|_{\alpha E}=\lambda_{\varphi}$ and $\left.\overline{\mu_{\varphi}}\right|_{E \alpha}=\mu_{\varphi}$, it follows from (1), (2) that $\left.\overline{\lambda_{\varphi}}\right|_{E \alpha}=\overline{\mu_{\varphi}}$ and $\left.\overline{\mu_{\varphi}}\right|_{\alpha E}=\lambda_{\varphi}$ so that $\Gamma^{+}(\varphi)=\Psi\left(\lambda_{\varphi}, \pi_{\lambda_{\varphi}, \mu_{\varphi}}, \mu_{\varphi}\right)$ as asserted. 
COROLlary. $\varphi \in$ Con $\alpha E \alpha$ is special if and only if $\varphi$ can be extended to a congruence on $S$.

Definition. We shall say that $\lambda \in$ Con $\alpha E$ is unitary if, in the quotient band $\alpha E / \lambda$, the class $[\alpha] \lambda$ is an identity element.

THEOREM 13. The following statements concerning $\lambda \in$ Con $\alpha E$ are equivalent:

(1) $\lambda$ is unitary;

(2) $\left.(\forall a, b \in \alpha E)(a \alpha, b \alpha) \in \lambda\right|_{\alpha E} \Rightarrow(a, b) \in \lambda$.

Proof. (1) $\Rightarrow(2)$. If $\lambda$ is unitary then $[a \alpha] \lambda=[a] \lambda[\alpha] \lambda=[a] \lambda$.

$(2) \Rightarrow(1)$. If (2) holds then

$$
\left.(b, a \alpha) \in \lambda \Rightarrow(b \alpha, a \alpha) \in \lambda\right|_{\alpha E \alpha} \Rightarrow(b, a) \in \lambda
$$

so that $[a \alpha] \lambda \subseteq[a] \lambda$ whence we have equality.

COROLlARY. The set of unitary congruences on $\alpha E$ is a sublattice of Con $\alpha E$.

Proof. If $\lambda, \lambda^{\prime} \in$ Con $\alpha E$ are unitary then it is clear from Theorem 13 that so also is $\lambda \cap \lambda^{\prime}$. If now $(a \alpha, b \alpha) \in \lambda \vee \lambda^{\prime}$ then there exist $z_{1}, \ldots, z_{n} \in \alpha E$ such that

$$
a \alpha \equiv z_{1} \equiv z_{2} \equiv \ldots \equiv z_{n} \equiv b \alpha
$$

where in each case $\equiv$ signifies either $\lambda$ or $\lambda^{\prime}$. It follows that

$$
a \alpha \equiv z_{1} \alpha \equiv z_{2} \alpha \equiv \ldots \equiv z_{n} \alpha \equiv b \alpha
$$

and therefore, by Theorem 13, that

$$
a \equiv z_{1} \equiv z_{2} \equiv \ldots \equiv z_{n} \equiv b .
$$

Consequently, $(a, b) \in \lambda \vee \lambda^{\prime}$ and so $\lambda \vee \lambda^{\prime}$ is also unitary.

Our interest in unitary congruences stems from the fact that if $\varphi \in \operatorname{Con} \alpha E \alpha$ then $\lambda_{\varphi}$ is unitary, as can be seen from Theorem 13 and the equivalences

$$
(a \alpha, b \alpha) \in \lambda_{\varphi} \Leftrightarrow(a \alpha, b \alpha) \in \varphi \Leftrightarrow(a, b) \in \lambda_{\varphi}
$$

THEOREM 14. If $\lambda \in \operatorname{Con} \alpha E$ and $\varphi=\left.\lambda\right|_{\alpha E \alpha}$ then $\lambda_{\varphi}$ is the smallest unitary congruence on $\alpha E$ that contains $\lambda$.

Proof. Observe that

$$
\left.(a, b) \in \lambda \Rightarrow(a \alpha, b \alpha) \in \lambda\right|_{\alpha E \alpha}=\varphi \Rightarrow(a, b) \in \lambda_{\varphi},
$$

so we have $\lambda \subseteq \lambda_{\varphi}$. If now $\rho$ is a unitary congruence on $\alpha E$ such that $\lambda \subseteq \rho$ then

$$
(a, b) \in \lambda_{\varphi} \Rightarrow(a \alpha, b \alpha) \in \varphi=\left.\left.\lambda\right|_{\alpha E \alpha} \subseteq \rho\right|_{\alpha E \alpha} \Rightarrow(a, b) \in \rho,
$$

so that $\lambda_{\varphi} \subseteq \rho$.

We define a unitary congruence $\mu \in \operatorname{Con} E \alpha$ in a similar way. By abuse of language, we shall say that $\vartheta \in$ Con* $S$ is unitary if both $\left.\vartheta\right|_{\alpha E}$ and $\left.\vartheta\right|_{E \alpha}$ are unitary. We shall denote by Con* $S$ the set of unitary congruences on $S$. 
If $\vartheta, \vartheta^{\prime} \in \operatorname{Con} S$ then it is clear that $\left.\left(\vartheta \cap \vartheta^{\prime}\right)\right|_{\alpha E}=\left.\left.\vartheta\right|_{\alpha E} \cap \vartheta^{\prime}\right|_{\alpha E}$. If now $(a, b) \in$ $\left.\left(\vartheta \vee \vartheta^{\prime}\right)\right|_{\alpha E}$ then there exist $z_{1}, \ldots, z_{n}$ such that

$$
a \equiv z_{1} \equiv z_{2} \equiv \ldots \equiv z_{n} \equiv b
$$

where each $\equiv$ signifies either $\vartheta$ or $\vartheta^{\prime}$. It follows that

$$
a=\alpha a \equiv \alpha z_{1} \equiv \alpha z_{2} \equiv \ldots \equiv \alpha z_{n} \equiv \alpha b=b
$$

and therefore $\left.\left.(a, b) \in \vartheta\right|_{\alpha E} \vee \vartheta^{\prime}\right|_{\alpha E}$. Hence we have that $\left.\left.\left.\left(\vartheta \vee \vartheta^{\prime}\right)\right|_{\alpha E} \subseteq \vartheta\right|_{\alpha E} \vee \vartheta^{\prime}\right|_{\alpha E}$, whence we have equality since the reverse inclusion is trivial. It now follows from the Corollary to Theorem 13 that $\operatorname{Con}_{1}^{*} S$ is a sublattice of Con* $S$.

Observe that if $\Psi(\lambda, \vartheta, \mu) \in$ Con $^{*} S$ and $\varphi=\left.\lambda\right|_{\alpha E \alpha}=\left.\mu\right|_{\alpha E \alpha}$ then it follows from Theorem 14 that $\Psi\left(\lambda_{\varphi}, \vartheta, \mu_{\varphi}\right) \in \operatorname{Con}_{1}^{*} S$ and the mapping $\Psi(\lambda, \vartheta, \mu) \mapsto \Psi\left(\lambda_{\varphi}, \vartheta, \mu_{\varphi}\right)$ is a closure on Con* $S$.

Although a description of the lattice Con* $S$ appears to be very difficult, we can describe the sublattice Con ${ }_{1}^{*} S$. For this purpose, we denote by Con $H_{\alpha}|\times| \operatorname{SpCon} \alpha E \alpha$ the set

$$
\left\{(\vartheta, \varphi) \in \operatorname{Con} H_{\alpha} \times \operatorname{SpCon} \alpha E \alpha ; \vartheta \subseteq \pi_{\lambda_{\varphi}, \mu_{\vartheta}}\right\} .
$$

THEOREM 15. $\operatorname{Con}_{1}^{*} S=\operatorname{Con} H_{\alpha}|\times| \operatorname{SpCon} \alpha E \alpha$.

Proof. For every $\varphi \in \mathrm{SpCon} \alpha E \alpha$ we have, by Theorem 12 and the fact that $\lambda_{\varphi}, \mu_{\varphi}$ are unitary,

$$
\Gamma^{+}(\varphi)=\Psi\left(\lambda_{\varphi}, \pi_{\lambda_{\varphi}, \mu_{\varphi}}, \mu_{\varphi}\right) \in \operatorname{Con}_{1}^{*} S .
$$

It follows by Theorem 8 that for $(\vartheta, \varphi) \in \operatorname{Con} H_{\alpha}|\times| \operatorname{SpCon} \alpha E \alpha$ we have

$$
\Psi\left(\lambda_{\varphi}, \vartheta, \mu_{\varphi}\right) \in \operatorname{Con}_{1}^{*} S .
$$

We can therefore define a mapping $\zeta$ :Con $H_{\alpha}|\times| \operatorname{SpCon} \alpha E \alpha \rightarrow \operatorname{Con}_{1}^{*} S$ by the prescription

$$
\zeta(\vartheta, \varphi)=\Psi\left(\lambda_{\varphi}, \vartheta, \mu_{\varphi}\right)
$$

Suppose now that $\Psi(\lambda, \vartheta, \mu) \in \operatorname{Con}_{1}^{*} S$. If $\varphi=\left.\lambda\right|_{\alpha E \alpha}=\left.\mu\right|_{\alpha E \alpha}$ then since $\lambda$ and $\mu$ are unitary we have $\lambda=\lambda_{\varphi}$ and $\mu=\mu_{\varphi}$; for example, by Theorem 13,

$$
(a, b) \in \lambda_{\varphi} \Leftrightarrow(a \alpha, b \alpha) \in \varphi=\left.\lambda\right|_{\alpha E \alpha} \Leftrightarrow(a, b) \in \lambda .
$$

Since $\vartheta \subseteq \pi_{\lambda, \mu}=\pi_{\lambda_{\varphi}, \mu_{\varphi}}$ we therefore have $(\vartheta, \varphi) \in \operatorname{Con} H_{\alpha}|\times| \operatorname{SpCon} \alpha E \alpha$. Consequently we can define a mapping $\eta: \operatorname{Con}_{1}^{*} S \rightarrow \operatorname{Con} H_{\alpha}|\times| \operatorname{SpCon} \alpha E \alpha$ by the prescription

$$
\eta \Psi(\lambda, \vartheta, \mu)=(\vartheta, \varphi)
$$

Now each of $\zeta, \eta$ is isotone; and we have

$$
\begin{aligned}
\eta \zeta(\vartheta, \varphi) & =\eta \Psi\left(\lambda_{\varphi}, \vartheta, \mu_{\varphi}\right)=(\vartheta, \varphi) ; \\
\zeta \eta \Psi(\lambda, \vartheta, \mu) & =\zeta(\vartheta, \varphi)=\Psi\left(\lambda_{\varphi}, \vartheta, \mu_{\varphi}\right)=\Psi(\lambda, \vartheta, \mu) .
\end{aligned}
$$

Thus $\eta, \zeta$ are mutually inverse isomorphisms. 
ACKNOWLEDGMENTS. NATO Collaborative Research Grant 910765 is gratefully acknowledged. The second-named author also gratefully acknowledges support from the Calouste Gulbenkian Foundation, Lisbon.

\section{REFERENCES}

1. T. S. Blyth, Emília Giraldes and M. Paula O. Marques-Smith, Associate subgroups of orthodox semigroups, Glasgow Math. J. 36 (1994), 163-171.

2. T. S. Blyth and M. F. Janowitz, Residuation theory (Pergamon Press, 1972). $39-42$.

3. T. S. Blyth and R. McFadden, Unit orthodox semigroups, Glasgow Math. J. 24 (1983),

T. S. BLYTH:

Mathematical InstituTe

UNIVERSITY OF ST ANDREWS

SCOTLAND

M. P. O. Marques-Smith:

Departamento de Matemática

UNIVERSIDADE Do Minho

Portugal
E. GiRALDES:

Departamento de Matemática

F.C.T., Universidade Nova de Lisboa

Portugal 